\title{
What sampling is needed for reliable estimations of genetic diversity in Fraxinus excelsior L. (Oleaceae)?
}

\author{
Naoko MIYAMOTO ${ }^{1,2}$, Juan F. FernándeZ-MANJARRÉs ${ }^{1 *}$, Marie-Elise MorAND-PRIEUR ${ }^{1,3}$, \\ Paola BERTOLINO ${ }^{1,4}$, Nathalie FrASCARIA-LACOSTE ${ }^{1,3}$ \\ ${ }^{1}$ Université Paris-Sud 11, Laboratoire Écologie, Systématique et Évolution, UMR 8079, 91405, Orsay, France \\ ${ }^{2}$ Present address: Forest Tree Breeding Center, Forestry and Forest Products Research Institute, 3809-1, Ishi Juo Hitachi Ibaraki 319-1301, Japan \\ ${ }^{3}$ AgroParisTech, 91405, Orsay, France \\ ${ }^{4}$ CNRS, 91405, Orsay, France
}

(Received 10 July 2007; accepted 29 January 2008)

\begin{abstract}
-
- Sample size is a critical issue for genetic diversity studies and conservation programs. However, sample size evaluation requires previous knowledge of allele frequencies estimated with precision and this is not often the case.

- Here, we evaluated sample size requirements for accurate genetic diversity in adult trees and family arrays in a 12 ha plot of Fraxinus excelsior L. (Oleaceae) in a community forest in central France. Data consisted of 579 adult trees and 480 offspring from 24 families genotyped at four nuclear microsatellites.

- Mean square errors (MSE) estimates performed on Monte Carlo simulations of resampled data indicated that several adult individuals (> 300) are necessary for accurate measures of allele richness. However, expected heterozygosity requires smaller samples $(<30)$. Seeds captured about $90 \%$ of adult allelic diversity requiring a sampling effort roughly 50\% larger than that of adult trees (480 seeds vs. 300 adults) suggesting that seed sampling is heavily penalized for allele counts. Nevertheless, gene diversity of seeds was essentially identical to that of the adult population.

- Extrapolation of these results to other ash tree populations appears feasible because of similar levels of diversity reported in the literature but it is not granted for species with significant selfing or high genetic structure.
\end{abstract}

Fraxinus excelsior / microsatellite/ genetic variation/ sampling

Résumé - Quel échantillonnage pour des estimations fiables de la diversité génétique chez Fraxinus excelsior L. (Oleaceae)?

- La taille d'un échantillonnage est un paramètre difficile à estimer a priori pour les études de diversité génétique ainsi que pour les programmes de conservation. En général, l'évaluation de cette taille nécessite au préalable une connaissance fine des fréquences alléliques ce qui n'est pas toujours le cas.

- Dans cette étude, nous avons évalué sur une parcelle de 12 ha de Fraxinus excelsior L. (Oleaceae), dans un forêt domaniale, la taille de l'échantillonnage nécessaire pour estimer de façon fiable la diversité génétique des arbres adultes ainsi que de leurs descendants. Un échantillon de 579 individus adultes et 480 graines issues de 24 arbres-mères ont été génotypés grâce à quatre marqueurs microsatellites nucléaires.

- Des analyses d'erreurs quadratiques moyennes obtenues dans le cadre de simulations de type Monte Carlo indiquent que plus de 300 individus adultes sont nécessaires pour obtenir des mesures alléliques fiables. Par contre, l'hétérozygotie espérée est obtenue pour des échantillons plus petits (<30). Les graines capturent $90 \%$ de la diversité allélique des adultes indiquant que l'échantillonnage des graines doit être deux fois celui des adultes pour obtenir la même information (480 graines vs. 300 adultes). Par ailleurs, la diversité génétique est identique pour les deux échantillonnages.

- L'extrapolation de ces résultats à d'autres espèces de frêne est possible compte tenu des niveaux de diversité observés dans la littérature mais n'est pas garantie pour des espèces qui s'autofécondent et qui ont des populations très structurées génétiquement.

Fraxinus excelsior / microsatellite / diversité génétique / échantillonnage

\section{INTRODUCTION}

Setting priorities for conservation of genetic resources is usually achieved by the genotyping of samples from various populations with one or more type(s) of neutral molecular markers e.g. (Petit et al., 1998). In particular, genotyping with microsatellite markers has become popular in population diversity studies (Zane et al., 2002) having frequently replaced less variable isozymes, and preferred in many cases over AFLP data because of codominance. For the case of plant

*Corresponding author: juan.fernandez@u-psud.fr species, whose sampling can be performed on either adults, offspring, or both, the question of allocating sampling effort is not easy to answer. Indeed, plant populations are now frequently analyzed simultaneously for mating system, parentage analyses or pollen dispersal curves (Austerlitz et al., 2004; Kalinowski et al., 2007; Ritland, 2002) so sampling sizes are becoming increasingly large. General wisdom based on parametric statistics suggests that 30 random individuals from a population should suffice for accurately estimating the sample mean of a given parameter (Petit et al., 1998; Sjogren and Wyoni, 1994). Indeed, most plant population studies have on 
average 50 individuals per population as a standard sample (Nybom, 2004), assuming that such samples suffice for an accurate estimation of all the desired parameters.

Sample size is also a concern in conservation genetics aimed at conserving adaptive variation in addition to neutral variation (Falk and Holsinguer, 1991; Lande and Barrowclough, 1987). However, the link between neutral and adaptive variation is difficult to assess in most cases (Holderegger et al., 2006). Nevertheless recent reviews (Kohn et al., 2006; Savolainen and Pyhajarvi, 2007) insist that neutral genetic diversity is still needed to tease apart historical and demographic processes from adaptive variation signals, which highlights the need for sound sampling designs for intra and inter population diversity measures. Among the different genetic parameters utilized in conservation genetics for pinpointing differences among populations or setting conservation priorities, allelic richness and gene diversity (expected heterozygosity) are usually considered key. Allele diversity directly reflects the raw material for evolution and gene diversity measures give the benefit of generality for purposes of comparison. In particular, standardized measures of allelic richness (Petit et al., 1998) have become widely utilized as a conservation criterion and methods are continuously refined to allow for inter-population comparisons (Van Loon et al., 2007) and private alleles detection in hierarchical sampling (Kalinowski, 2004).

Several attempts have been made to estimate sample sizes appropriate for genetic diversity parameter estimation e.g. (Cavers et al., 2005), but all methods require a previous knowledge of genotype frequencies for a given population. For instance, Ohsawa and collaborators (Ohsawa et al., 2007) have noted recently that the lack of adult spatial genetic structure in some of their plots of Quercus crispula could be due to small sample size $(<50)$ but they could not verify this. In consequence, sampling schemes can only be validated a posteriori with the intention of guiding future sampling programs. It follows then that intensively studied populations with at least samples of 500 individuals or more are extremely valuable for evaluating sampling strategies because the precision of estimates should be reliable beyond any computational doubts. Thus, using detailed information from an intensively studied plot in a large stand of Fraxinus excelsior in France (MorandPrieur, 2003), we evaluated, through resampling, the impact of sample size on the accuracy of genetic diversity parameters. In particular, we asked the following questions: (1) How many adult trees should be sampled to adequately represent the genetic diversity of a common ash population? (2) What is the optimal combination of number of maternal trees and seeds per tree when sampling family arrays? In other words, how many seeds from how many trees are needed to adequately sample the genetic diversity of the adult population?

\section{MATERIALS AND METHODS}

\subsection{Study species}

Fraxinus excelsior L. (Oleaceae), the common ash, is valued as a timber tree because of its rapid growth as well as its tough and elastic wood. In addition, the interest of this species in reforestation programs has increased over the last decade. The common ash has many ecological traits that can be associated with high levels of genetic diversity (Hamrick and Godt, 1996): it is a colonizing species with a large but discontinuous distribution (Franc and Ruchaud, 1996), it is wind-pollinated and produces wind dispersed fruits (Wardle, 1961). In addition, this species has various sex types from completely male individuals to pure female individuals, with various types of hermaphrodites in between (Morand-Prieur, 2003). Finally, it exhibits practically no selfing (Morand-Prieur, 2003), a rather weak population structure and long distance pollen dispersal (Miyamoto et al. in preparation).

\subsection{Sampling}

We sampled leaves from 579 individuals from the contiguous population at the forest in Dourdan (surface plot 12 ha, altitude: $130 \mathrm{~m}$, longitude $02^{\circ} 00^{\prime} 42^{\prime \prime}$, latitude $48^{\circ} 31^{\prime}$ ' 47’; Essonne) in France in 1999 for an exhaustive genotyping of the adult population. Next, we chose 24 focal trees (mean separation of $248 \mathrm{~m}, \mathrm{SD}=159 \mathrm{~m}$ ) from this adult population for a mating system analysis that we treat elsewhere (Morand-Prieur, 2003) sampling 20 seeds per tree resulting in 480 total offspring. Finally, we included an additional 20 seeds from 10 of the above trees to examine sampling behavior of the most variable locus (see below) resulting in a second group of family arrays of 400 total size (10 trees, 40 seeds per tree).

\subsection{Molecular techniques}

DNA extraction for all the samples was performed using the Dneasy ${ }^{\circledR}$ Plant Mini Kit (Quiagen). We genotyped the above mentioned samples using four microsatellite loci: M230 from (Brachet et al., 1999), and FEMSATL4, FEMSATL11 FEMSATL16 from (Lefort et al., 1999). The segregation of alleles for these markers has already been validated using control-pollinated full-sibs and no deviation from Mendelian segregation or null alleles were detected (Morand-Prieur, 2003). Polymerase chain reaction was performed as described in (Brachet, et al., 1999). PCR products were separated on an $8 \%$ polyacrilamide gel, and visualized using the FM-BIO II (Hitachi) scanner. Alleles were scored by comparison with certain trees whose genotypes were sequenced for exact allele size.

\subsection{Adult genetic diversity evaluation}

We used resampling simulations to estimate the sampling effort required for an accurate estimation of various genetic parameters. Herein, we define accuracy as the difference between an estimate and the true value of the population, here estimated from the largest sample available, and precision as the variance of the estimates (Hellmann and Fowler, 1999). In consequence, accuracy is measured with a bias estimator, and precision with standard errors and related measures. We combined the methods proposed by (Banks et al., 2000) to measure allelic diversity by Monte Carlo resampling and those by (Kirst et al., 2005) to measure the accuracy of the estimations through the use of mean square errors (MSE). Therefore, resampling without replacement from the whole adult population $(N=579)$ was performed for samplings efforts of $n=1,2,3, \ldots, 579$, repeating the procedure 1000 times at each sampling size. 
We estimated the mean of the simulations for each sample size and determined the bias by comparing this mean with the total population mean. The bias of the estimators was evaluated by joint analysis of the coefficient of variation and the mean square error following Kirst et al. (2005). In this framework, the bias is the difference between the expected value of the estimator (the mean of the estimates of all possible samples that can be taken from the population) and the true population value (Hellmann and Fowler, 1999). Hence, we used the formula:

$$
C V_{M S E i}=\frac{\sqrt{\left(\bar{x}_{i}-\Theta\right)^{2}+\frac{1}{b} s_{i}^{2}}}{\bar{x}_{i}}
$$

where $\bar{x}_{i}$ and $s_{i}^{2}$ represent the mean and the standard error (Monte Carlo mean and standard deviation) of the different estimations for sample size $i$, respectively. The first term of the numerator $\left(\bar{x}_{i}-\Theta\right)$ represents the bias between the "true" value of the parameter $\Theta$ and the estimate for a given sample size, and $b$ is the number of iterations for a given sample size $(b=1000)$. When the bias is negligible, the $C V_{m s e}$ is simply the coefficient of variation (CV). Finally, the sample size necessary for achieving a $C V_{m s e}$ equal to or smaller than 0.10 , 0.05 and 0.01 (or 0.001 ) was recorded. The advantage of this approach is that even if we do not see the individual behavior of the mean and the variance one can verify if both parameters have stabilized because they are summarized in the CV. We inspected visually during preliminary runs to verify that the threshold values that we used were effectively in the zone where the $C V_{\text {mse }}$ is already asymptotic indicating that both the variance and the mean of the estimates had stabilized.

We estimated the number of alleles $A$ by direct counting. The number of effective alleles $A_{\mathrm{e}}$ (a measure of the maximum possible diversity if all alleles had the same frequency) was calculated as $1 / \Sigma p_{i}^{2}$ were $p_{i}$ is the frequency of the $i$ th allele. Observed heterozygosity $H_{o}$ was estimated by direct counting of heterozygote individuals, and expected genetic diversity $H_{\mathrm{e}}$ with the formula of Nei and Roychoudhury (1974) that accounts for sample size bias and inbreeding in the population $H_{e}=\frac{n}{n-1}\left[1-\sum_{i} p_{i}^{2}-\frac{H_{o}}{2 n}\right]$. Again, $p_{i}$ is the frequency of the $i$ th allele, $H_{o}$ is the observed heterozygosity and $n$ is the sample size. Finally the inbreeding coefficient $f_{i s}$ was estimated as $f_{i s}=1-H_{o} / H_{\mathrm{e}}$. All estimates were averaged across loci. We concentrated on the following genetic parameters: total number of alleles (allelic richness), effective number of alleles and expected heterozygosity. Even though exact variances are known for expected diversity e.g. (Nei, 1987; Weir, 1995) for fixed sized populations, to the best of our knowledge, no exact solutions exist for the resampling of alleles, or for genetic diversity for that matter. Hence, we relied on the Monte Carlo methods outlined here.

\subsection{Family arrays diversity evaluation}

Similarly, for the family arrays ( 24 families of 20 seeds each), we performed a resampling simulation as for the total population. We sampled without replacement 1,2, $3 \ldots, 24$ trees and within each simulation $1,2,3 \ldots, 20$ seeds repeating the procedure 1000 times. Next, all results were averaged and the standard error calculated and we applied equation 1 . Results were then displayed in a $20 \times 24$ grid and contour lines of the genetic parameters and of $C V_{\text {mse }}$ values of $0.10,0.05$ and 0.01 were drawn. Bias was estimated separately with reference to: a) the sampled array population (480 seeds); and b) to the standardized value of the adult population at $n=400$ and $n=480$.

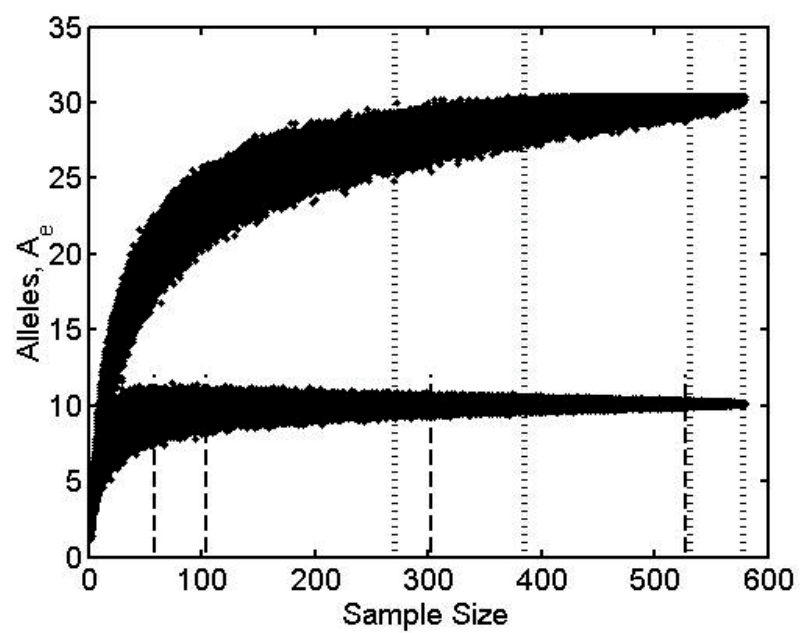

Figure 1. Resampling simulation of the adult population of common ash from the Dourdan forest in France. Results are from 1000 iterations at each sample size ( $n=1$ to 579), including allele richness (upper points) and effective number of alleles (lower points). Thresholds for $C V_{m s e}$ of $0.10,0.05,0.01$ and 0.001 are presented as fine dots for the allelic counts (on the right) and as dashes for effective number of alleles on the left. Curves converge to the population value.

For this last standardized value we used the results from the first simulations, as resampling without replacement for different sizes produce the same results as rarefaction techniques (Petit et al., 2005), but with the advantage of providing standard errors for the estimations (Fernández-Manjarrés, unpublished results). To further investigate the effect of tree vs. family sampling efforts, we genotyped a subset of 10 of the original 24 families with the most variable molecular marker M2-30 for 40 seeds per tree $(n=40)$. We assumed that any patterns should be visible for the most variable locus and results for this locus could be used as a guide for the general sampling. All simulations were written in Matlab ${ }^{\circledR} \mathrm{R} 12$ and are available upon request from JFFM.

\section{RESULTS}

\subsection{Total population sampling}

The resampling of the total population suggests that relatively large samples are required to capture most of the mean number of alleles present in the adult population with good accuracy: 272, 386 and 532 adult trees are necessary if a $C V_{m s e}$ 's of $0.10,0.05$ and 0.01, respectively, are desired (Fig. 1). Similarly, 59, 104, 303 adult trees are necessary for similar $C V_{m s e}$ 's of the effective number of alleles $A_{\mathrm{e}}$.

In contrast, very small sample sizes are necessary to obtain small $C V_{\text {mse }}$ 's of the expected heterozygosity as the mean of the simulations are close to the population value at all sample sizes (small bias), although the variance was larger for samples of fewer than 50 trees (Fig. 2). In fact, $C V_{m s e}$ 's of 0.01 are possible with a sample size of $n=4$, so a more realistic criterion for gene diversity is probably 0.001 that requires 31 individuals on average. 


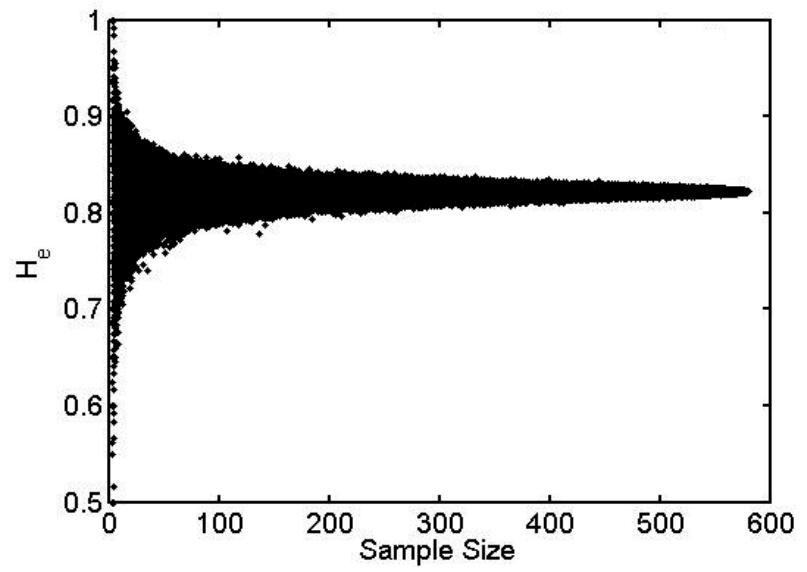

Figure 2. Resampling simulation of the adult population of common ash from the Dourdan forest in France. Results are from 1000 iterations at each sample size ( $n=1$ to 579 ) for genetic diversity (expected heterozygosity). $C V_{m s e}$ 's thresholds are fairly small and not depicted (size for $0.001<30$ ). The curve converges to the population value.

In general for this adult population, sampling variance of allele counts is much larger than for the rest of the parameters (Tab. I) resulting in the need for large sample sizes for obtaining accurate estimates (close to the true value). On the other hand, observed and expected genetic diversity exhibit good accuracy, but samples below 30 individuals have low precision (large variance) as seen in figure 2 for $H_{\mathrm{e}}$ and for $H_{\mathrm{o}}$ (results not shown). The inbreeding coefficient appears to fall in between, requiring sample sizes intermediate between those required for allele counts and heterozygosities (Tab. I). Hereafter we will concentrate on allele diversity and expected heterozygosity for both the adults and family arrays.

\subsection{Standardized genetic diversity}

The resampling of 480 adult individuals indicated that the standardized (rarefacted) number of alleles for the four loci evaluated was $A=30.8$ (95\% CI: $30.0-31.5), A_{\mathrm{e}}=9.9(95 \%$ CI: 9.7-10.1) and $H_{\mathrm{e}}=0.7996$ (95\% CI: 0.7947-0.8044). For the most variable locus, M2-30 the standardized diversity for $n=400$ was $A=46.9$ (95\% CI: $45.0-48.0), A_{\mathrm{e}}=22.7$ (95\% CI: 21.6-23.9) and $H_{\mathrm{e}}=0.9560$ (95\% CI: 0.9537-0.9581). These values were used then for contrasting the observed diversity in the seed family arrays with the adult population as shown next.

\subsection{Family array sampling}

Again, relatively large sample sizes are necessary to obtain a good representation of allelic diversity in the offspring, but the sampling effort is less if more maternal trees are sampled, as expected. On average, two alleles per locus were missed, as the mean $A$ for the offspring arrays was 28.3 vs. $A=30.8$ (standardized adult value at 480 ). Hence, about $92 \%$ of the alleles were obtained with the predefined sampling. As seen in
Table I. Sample sizes of different genetic parameters (average of 5 loci) required to attain a given coefficient of variation of the mean square error $C V_{\text {mse }}$ for the adult population.

\begin{tabular}{lcccc}
\hline Parameter & \multicolumn{4}{c}{$C V_{\text {mse }}$} \\
\cline { 2 - 5 } & 0.1 & 0.05 & 0.01 & 0.001 \\
\hline$A^{1}$ & 272 & 386 & 532 & 578 \\
$A_{\mathrm{e}}$ & 59 & 104 & 303 & 527 \\
$H_{\mathrm{o}}$ & 3 & 3 & 4 & 77 \\
$H_{\mathrm{e}}$ & 4 & 4 & 4 & 31 \\
$f_{\text {is }}$ & 10 & 13 & 67 & 546 \\
\hline
\end{tabular}

${ }^{1} A$ : number of alleles; $A_{\mathrm{e}}$ : number of effective alleles; $H_{\mathrm{o}}$ observed heterozygosity; $H_{\mathrm{e}}$ : expected heterozygosity; and $f_{\text {is }}$ : inbreeding coefficient.

Figure $3 \mathrm{~A}$, the sampling effort for 25 alleles on average is only 9 seeds from each of 24 maternal trees $=216$, whilst 20 seeds from each of 14 maternal trees $=280$ are needed to get the same number of alleles. However, at least 312 offspring are necessary to get a $C V_{m s e}$ of 0.05 or less indicating that large samples are still necessary to stabilize the allele sampling variance for family arrays (Fig. 3B). In general, seed array sampling is less efficient for detecting diversity as 480 seeds sampled roughly the same allelic diversity than 300 random adults, suggesting that about $50 \%$ more seeds than adults are needed to get the same information.

The offspring array sampling yielded 9.3 effective alleles from a standardized value of 9.9 , so about $94 \%$ of the gene diversity was captured this way. Also, it is clear cut that it is preferable to sample more trees than more offspring per tree for both the number of effective alleles and the $C V_{m s e}$ (Figs. 4A and 4B). For example, only 96 samples are needed for a $C V_{m s e}$ of 0.05 if 24 trees are sampled (4 seeds per tree), compared to 300 samples for 15 maternal trees ( 20 seeds per tree). The same holds for 9 effective alleles (Fig. 4A). For the case of family arrays of size 40 , the results for the most variable marker (see below) also confirm that we are better served by sampling more trees than more seeds. For example, about 150 samples (10 trees, 15 offspring per tree) detect an equivalent number of effective alleles as 200 samples that come from 5 trees and 40 seeds each (results not shown).

Expected diversity on the other hand is much more forgiving, as seen from the total population analysis, and relatively few samples are needed to obtain accurate estimations (Fig. 5). It is clear from the $H_{\mathrm{e}}$ values (Fig. 5A) that one or two seeds from 24 trees suffice to accurately estimate the genetic diversity of the adult population from seed arrays ( 0.7895 vs. 0.7996 for the adults). In fact, offspring measures were within $C V_{\text {mse }}$ of 0.01 of the total adult population. The implemented sampling showed also that about 50 seeds will provide a $C V_{\text {mse }}$ of 0.01 (say 10 trees, 5 seeds per tree, Fig. 5B), and that very little is gained if more seeds per trees are sampled.

Finally, for the most variable locus, M2-30 (figures not shown), the 400 seeds issued from the subset of 10 trees provided 44 alleles from a standardized value of $A=46.9$, that is statistically significantly lower as judged by the $95 \%$. 


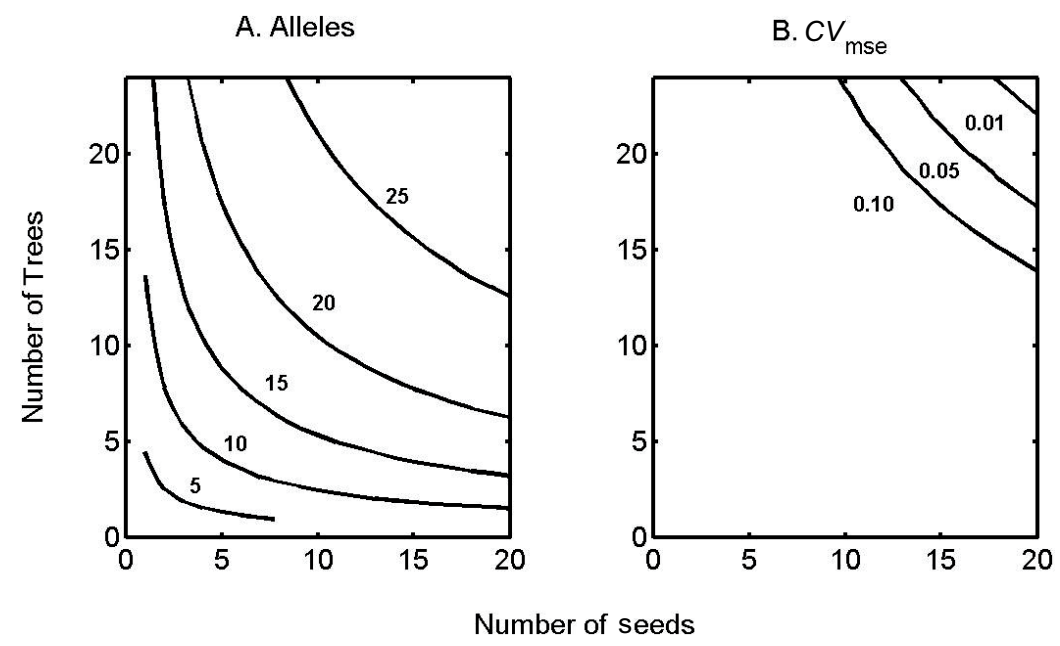

Figure 3. Resampling results for 24 family arrays of 20 seeds each for allelic richness. (A) Curves represent the combination of number of trees, number of seeds per tree yielding 5, 10, 15, 20, 25 alleles; (B) curves represent tree/seed combinations that produce $C V_{m s e}$ 's of $0.10,0.05$, 0.01. The $C V_{\text {mse }}$ depicted are calculated with reference to the array and not to the total adult population.

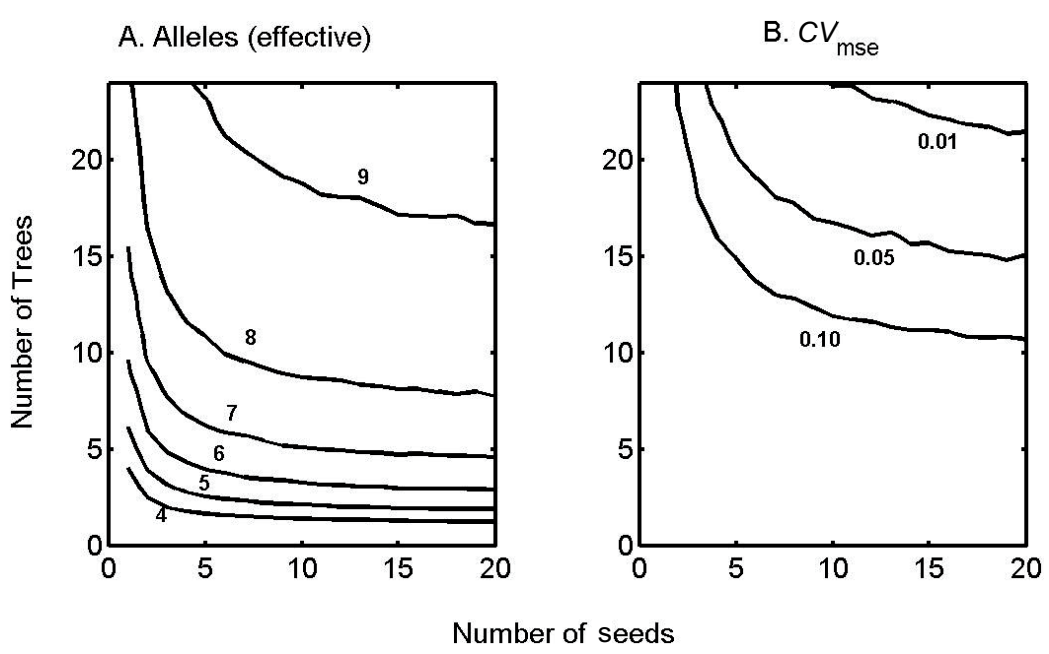

Figure 4. Resampling results for 24 family arrays of 20 seeds each for genetic diversity. (A) Curves represent the combination of number of trees, number of seeds per tree yielding $A_{\mathrm{e}}=4$ to 9 ; (B) curves represent tree/seed combinations that produce $C V_{m s e}$ 's of $0.10,0.05,0.01$.

On average, $94 \%$ of alleles were sampled which is in accord with previous results. The estimation of effective alleles was somewhat less efficient, as only 18.9 from 22.7 were detected $(83.4 \%)$. Lastly, the estimation of genetic diversity was sound as expected, as the 400 seeds yielded a $H_{\mathrm{e}}$ of 0.9486 , very close to the standardized value of $H_{\mathrm{e}}=0.9560$. These results indicate again that little is gained by doubling family array sizes, even for the most variable marker.

\section{DISCUSSION}

Most studies of genetic diversity in plant populations use moderate sample sizes of about 50 individuals per population (Nybom, 2004). However, our results show clearly that if nuclear microsatellites are used in F. excelsior, such "standard" sample will underestimate the allelic richness of the popula- tion. In fact, the resampling at size $n=50$ of the adult population showed that all 1000 iterations gave samples with 21 alleles or less (range 16 to 21, mean $=18.8$, numbers from Fig. 1) out of 31 possible alleles. Our results are similar to those for trout populations measured also with microsatellites whose sampling requirement is between 200 and 300 individuals if most of the alleles are to be captured (Banks, et al., 2000). If hypervariable microsatellites are the chosen tool for population diversity measures and allelic richness and inclusion of rare alleles the conservation goal, population sizes that need to be sampled and preserved will necessarily be large.

Effective allele estimation, on the other hand, indicates that for the same "standard" sample, the estimation is almost correct, requiring on average 56 individuals to obtain a $C V_{m s e}$ of 0.10 (Tab. I). This size can be thought as the minimum, as effective allele measures reflect the diversity of common alleles, which contribute most to diversity. Similarly, the precise 


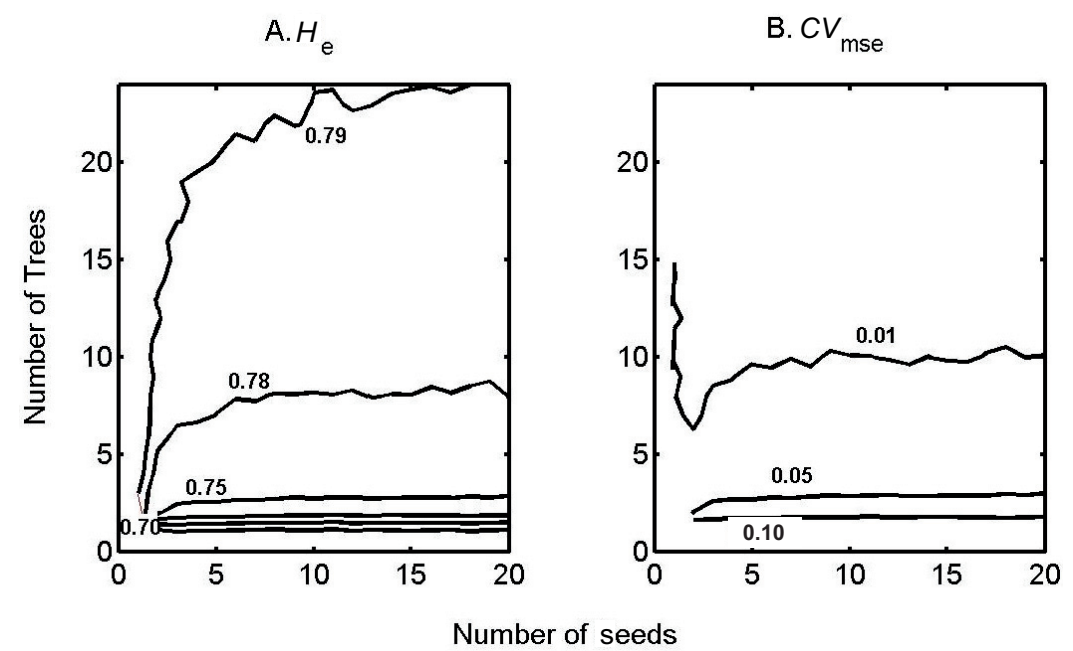

Figure 5. Resampling results for 24 family arrays of 20 seeds each for genetic diversity. (A) Curves represent the combination of number of trees, number of seeds per tree yielding diversities of $H_{\mathrm{e}}=0.75,0.78,0.79$; (B) curves represent tree/seed combinations that produce $C V_{m s e}$ 's of $0.10,0.05,0.01$.

estimation of genetic diversity is achieved with relatively small samples. Hence, it appears less error-prone to use the effective alleles and genetic diversity than allele counts for determining the diversity of a population. Current software that include rarefaction methods (see for example Goudet, 1995) help to avoid erroneous conclusions when comparing small and large populations, but doubts will remain about decreased diversity from populations sampled with fewer individuals.

How general are our results? At least for the case of the widespread common ash in Europe, genetic diversity results derived from nuclear microsatellite loci (Fernandez-Manjarres et al., 2006; Ferrazzini et al., 2007; Gerard et al., 2006; Hebel et al., 2006; Heuertz et al., 2001; Morand et al., 2002) suggest that allelic richness and gene diversity are fairly similar. Yet, more important than the numbers of alleles is the identity of those alleles and genetic conservation should favor the preservation of private alleles (Kalinowski, 2004; 2005), a result only possible if several populations are sampled. Similar conclusions should be applicable to outcrossing trees with similar levels of diversity and similar levels of inter-population structure (i.e., Fst < 0.05). It is uncertain whether we can use these results for the sampling program of other species, but these results would be useful if we consider species that have similar distribution (pan European), way of propagation (windpollinated, dispersion of the fruits by wind), and density (about 600 individuals in 12 ha) as common ash.

Would we get the same answer with other genetic markers? Clearly, if emphasis is put on allele richness the use of other genetic markers may lead to different conclusions. In fact, marker choice certainly complicates matters as shown in a recent analysis (Scotti et al., 2006) on Norway spruce (Picea abies) where population differentiation estimates are not only correlated to sample size but to marker choice (di and tri nucleotide nuclear SSR and mononucleotide chloroplast SSR). In the case of allozyme markers in ash or other species, with many fewer alleles than microsatellites, sample sizes will necessarily be smaller than those suggested here (300). In other words, the "standard" sampling of 50 individuals will largely suffice. Unfortunately, we do not have access to such type of markers in our common ash case.

Finally, a sampling scheme tailored to estimate general diversity is not necessarily inconsistent with one to estimate genetic structure. Our results show that it is equivalent to sample, say, 24 trees and four seeds per tree as 10 trees and 20 seeds per trees (Fig. 4). In fact, this principle of sampling several trees and few seeds per tree is perfectly acceptable and even desirable when analyzing pollen structure (Austerlitz and Smouse, 2002), provided gene flow is relatively homogeneous and that there is no serious suspicion of anisotropy in pollen or seed movement (F. Austerlitz, pers. comm.). As for mating system analysis in general, at least eight seeds per family array are needed to correctly infer the maternal genotype (K. Ritland, MLTR v. 3 manual) and this could be considered as a good starting point. Thus, we can conclude that future mating system studies in common ash can incorporate about 8 seeds per family array from 50 trees $(n=400$, identical to this study) that should capture adequate details on genetic diversity and mating patterns at the same time, probably sparing the need to sample large numbers of adults. Indeed, we simulated from one to 200 random families of size eight (not shown) and found that upwards of 150 families we would capture almost $100 \%$ of alleles from the adult population. Thus, a family array based sampling of total size $150 \times 8=1200$ would serve for diversity estimates and a better mating system and pollen dispersal analysis. Our original sampling had around 1000 samples consisting of almost 600 adults and 400 seeds, which now appears limited. Clearly, sampling several small families yields more information and in a more cost- and labor-efficient way. 
We have one main methodological concern about the impact of resampling without replacement over a finite population that generates covariation between different sample sizes and their Monte-Carlo variance. As sample size increases, samples share more individuals, reducing the Monte-Carlo variance between estimates as can be seen in Figures 1 and 2 . One way to circumvent this size dependency on the estimated variance is to assume that allele frequencies were adequately estimated (which is impossible to verify in most cases). Next, one could create a large population with Hardy-Weinberg inbreeding proportions upon which resampling is performed. In fact, we simulated a 100000 individual population as just described and we applied the same criteria as those used for the empirical data (results not shown). Our results on this "infinite" population indicated that 627 and 132 adult trees are needed to obtain a $C V_{\text {mse }}$ of 0.05 for the total number of alleles and effective alleles instead of 386 and 104, respectively. In contrast, fewer individuals are needed for obtaining a $C V_{\text {mse }}$ of 0.001 for the genetic diversity parameter: 20 instead of 31 . This simulation indicates that the Monte-Carlo variance of allele counts is more affected by covariance of sample size, while $A_{\mathrm{e}}$ and $H_{\mathrm{e}}$ are more robust. Indeed, the sample size suggested for $A_{\mathrm{e}}$ appears to be a good compromise between the large requirements for exhaustive allele counts and the small ones for accurate expected heterozygosity. Nevertheless, creating large populations to verify empirical sample sizes needs to be evaluated in other large population samples before generalizing this approximation.

In conclusion, our results show that for a wind pollinated species, whose biology favors high genetic diversity (no selfing, long distance pollen dispersal, long distance seed dispersal) large sampling efforts (about 300 adults or its equivalent issued from seeds from sufficiently spaced trees) are needed if an accurate estimation of allelic richness is the target of the study. Similarly, from a genetic resources perspective, large populations are needed to maintain the large allelic diversity (here judged by nuclear microsatellite markers) if we assume, as a first approximation, that adaptive diversity is as variable as the neutral counterpart. Measuring diversity by means of expected heterozygosity appears much less demanding and random samples from ash populations seem to be adequate for this purpose. As far as species with high selfing and/or strong assortative mating, sampling effort might be difficult to judge because population structure would be much higher than for common ash populations. Unfortunately, the retrospective analyses here presented can only be used a posteriori, and only long term programs can benefit from the verification of initial sampling schemes.

Acknowledgements: We would like to thank two anonymous reviewers who greatly helped to improve our manuscript and J. Shykoff for useful comments and grammar verification. We would also like to thank B. Jung-Muller and L. Nowak for their help in the field and C. Raquin for constructive discussion of the results. N. Miyamoto was supported by a of French Government grant from Egide. JF Fernández-Manjarrés was supported by a post-doctoral position funded by the EU, ME Morand acknowledges an FCPR grant from the French Ministry of Agriculture (FMA). Financial support was provided by the DGFAR project and the EC (RAP Project QLK52001600631).

\section{REFERENCES}

Austerlitz F., Dick C.W., Dutech C., Klein E.K., Oddou-Muratorio S., Smouse P.E., and Sork V.L., 2004. Using genetic markers to estimate the pollen dispersal curve. Mol. Ecol. 13: 937-954.

Austerlitz F. and Smouse P.E., 2002. Two-generation analysis of pollen flow across a landscape. IV. Estimating the dispersal parameter. Genetics 161: 355-363.

Banks M.A., Rashbrook V.K., Calavetta M.J., Dean C.A., and Hedgecock D., 2000. Analysis of microsatellite DNA resolves genetic structure and diversity of chinook salmon (Oncorhynchus tshawytscha) in California's Central Valley. Can. J. Fish. Aquat. Sci. 57: 915-927.

Brachet S., Jubier M.F., Richard M., Jung-Muller B., and FrascariaLacoste N., 1999. Rapid identification of microsatellite loci using 5, anchored PCR in the common ash Fraxinus excelsior. Mol. Ecol. 8: $160-163$.

Cavers S., Degen B., Caron H., Lemes M.R., Margis R., Salgueiro F., and Lowe A.J., 2005. Optimal sampling strategy for estimation of spatial genetic structure in tree populations. Heredity 95: 281-289.

Falk D.A. and Holsinguer K.E., 1991. Genetics and conservation of rare plants. Oxford University Press, New York, 283 p.

Fernandez-Manjarres J.F., Gerard P.R., Dufour J., Raquin C., and Frascaria-Lacoste N., 2006. Differential patterns of morphological and molecular hybridization between Fraxinus excelsior L. and Fraxinus angustifolia Vahl (Oleaceae) in eastern and western France. Mol. Ecol. 15: 3245-3257.

Ferrazzini D., Monteleone I., and Belletti P., 2007. Genetic variability and divergence among Italian populations of common ash (Fraxinus excelsior L.). Ann. For. Sci. 64: 159-168.

Franc A. and Ruchaud F., 1996. Le Frêne commun. In: CEMAGREF (Ed.), Autécologie des feuillus precieux: frêne commun, merisier, érable sycomore, erable plane, CEMAGREF, Gap, pp. 15-68.

Gerard P.R., Fernandez-Manjarres J.F., and Frascaria-Lacoste N., 2006. Temporal cline in a hybrid zone population between Fraxinus excelsior L. and Fraxinus angustifolia Vahl. Mol. Ecol. 15: 3655-3667.

Goudet J., 1995. FSTAT (Version 1.2): A computer program to calculate F-statistics. J. Hered. 86: 485-486.

Hamrick J.L. and Godt M.J.W., 1996. Effects of life history traits on genetic diversity in plant species. Philos. Trans. R. Soc. Lond B-Biol. Sci. 351: 1291-1298.

Hebel I., Haas R., and Dounavi A., 2006. Genetic variation of common ash (Fraxinus excelsior L.) populations from provenance regions in southern Germany by using nuclear and chloroplast microsatellites. Silvae Genet. 55: 38-44.

Hellmann J.J. and Fowler G.W., 1999. Bias, precision and accuracy of four measures of species richness. Ecol. Appl. 9: 824-834.

Heuertz M., Hausman J.F., Tsvetkov I., Frascaria-Lacoste N., and Vekemans X., 2001. Assessment of genetic structure within and among Bulgarian populations of the common ash (Fraxinus excelsior L.). Mol. Ecol. 10: 1615-1623.

Holderegger R., Kamm U., and Gugerli F., 2006. Adaptive vs. neutral genetic diversity: implications for landscape genetics. Landsc. Ecol. 21: 797-807.

Kalinowski S.T., 2004. Counting alleles with rarefaction: Private alleles and hierarchical sampling designs. Conserv. Genet. 5: 539-543. 
Kalinowski S.T., 2005. HP-RARE 1.0: a computer program for performing rarefaction on measures of allelic richness. Mol. Ecol. Notes 5: $187-189$

Kalinowski S.T., Taper M.L., and Marshall T.C., 2007. Revising how the computer program CERVUS accommodates genotyping error increases success in paternity assignment. Mol. Ecol. 16: 1099-1106.

Kirst M., Cordeiro C.M., Rezende G., and Grattapaglia D., 2005. Power of microsatellite markers for fingerprinting and parentage analysis in Eucalyptus grandis breeding populations. J. Hered. 96: 161-166.

Kohn M.H., Murphy W.J., Ostrander E.A., and Wayne R.K., 2006. Genomics and conservation genetics. Trends Ecol. Evol. 21: 629637.

Lande R. and Barrowclough G.F., 1987. Effective population size, genetic variation, and their use in population management., In: Soulé M.E. (Ed.), Viable populations for conservation, Cambridge University Press, Cambridge, pp. 87-124.

Lefort F., Brachet S., Frascaria-Lacoste N., Edwards K.J., and Douglas G.C., 1999. Identification and characterization of microsatellite loci in ash (Fraxinus excelsior L.) and their conservation in the olive family (Oleaceae). Mol. Ecol. 8, 1088-1090.

Morand-Prieur M.E., 2003. Évolution et maintien d'un système de reproduction polymorphe. Approche génétique et écologique de la polygamie chez le frêne commun, Fraxinus excelsior L., ENGREF, Paris, $95 \mathrm{p}$.

Morand M.E., Brachet S., Rossignol P., Dufour J., and Frascaria-Lacoste N., 2002. A generalized heterozygote deficiency assessed with microsatellites in French common ash populations. Mol. Ecol. 11: 377385

Nei M., 1987. Molecular Evolutionary Genetics, Columbia University Press, New York, 512 p.

Nei M. and Roychoudhury A.K., 1974. Sampling variances of heterozygosity and genetic distance. Genetics 76: 379-390.
Nybom H., 2004. Comparison of different nuclear DNA markers for estimating intraspecific genetic diversity in plants. Mol. Ecol. 13: 11431155.

Ohsawa T., Tsuda Y., Saito Y., Sawada H., and Ide Y., 2007. Steep slopes promote downhill dispersal of Quercus crispula seeds and weaken the fine-scale genetic structure of seedling populations. Ann. For. Sci. 64: 405-412.

Petit R.J., Deguilloux M.F., Chat J., Grivet D., Garnier-Gere P., and Vendramin G.G., 2005. Standardizing for microsatellite length in comparisons of genetic diversity. Mol. Ecol. 14: 885-890.

Petit R.J., El Mousadik A., and Pons O., 1998. Identifying populations for conservation on the basis of genetic markers. Conserv. Biol. 12: 844-855.

Ritland K., 2002. Extensions of models for the estimation of mating systems using $\mathrm{n}$ independent loci. Heredity 88: 221-228.

Savolainen O. and Pyhajarvi T., 2007. Genomic diversity in forest trees. Curr. Opin. Plant Biol. 10: 162-167.

Scotti I., Paglia G., Magni F., and Morgante M., 2006. Population genetics of Norway spruce (Picea abies Karst.) at regional scale: sensitivity of different microsatellite motif classes in detecting differentiation. Ann. For. Sci. 63: 485-491.

Sjogren P. and Wyoni P.I., 1994. Conservation Genetics and Detection of Rare Alleles in Finite Populations. Conserv. Biol. 8: 267-270.

Van Loon E.E., Cleary D.F.R., and Fauvelot C., 2007. ARES: software to compare allelic richness between uneven samples. Mol. Ecol. Notes 7: $579-582$.

Wardle P., 1961. Biological flora of the British Isles. Fraxinus excelsior L. J. Ecol. 49: 739-751.

Weir B.S., 1995. Genetic Data Analysis II, Sinauer Associates, Inc. Publishers, Sunderland, 445 p.

Zane L., Bargelloni L., and Patarnello T., 2002. Strategies for microsatellite isolation: a review. Mol. Ecol. 11: 1-16. 